\title{
Phase I study of the indoleamine 2,3-dioxygenase 1 inhibitor navoximod (GDC-0919) as monotherapy and in combination with the PD-L1 inhibitor atezolizumab in Japanese patients with advanced solid tumours
}

\author{
Takahiro Ebata $^{1}$ - Toshio Shimizu ${ }^{1}$ - Yutaka Fujiwara ${ }^{1} \cdot$ Kenji Tamura $^{2} \cdot$ Shunsuke Kondo $^{1} \cdot$ Satoru Iwasa $^{1}$. \\ Kan Yonemori ${ }^{1}$ - Akihiko Shimomura ${ }^{1}$. Shigehisa Kitano ${ }^{1}$ - Takafumi Koyama ${ }^{1}$ - Natsuko Sato ${ }^{3}$. Kiyohiko Nakai ${ }^{3}$. \\ Michiyasu Inatani ${ }^{3} \cdot$ Noboru Yamamoto $^{1}$
}

Received: 1 March 2019 / Accepted: 25 April 2019/Published online: 24 May 2019

(C) The Author(s) 2019

\section{Summary}

Navoximod (GDC-0919) is a small molecule inhibitor of indoleamine-2,3-dioxygenase 1. This study investigated the safety, tolerability and pharmacokinetics of navoximod alone and in combination with atezolizumab in Japanese patients with advanced solid tumours. This was a phase I, open-label, dose-escalation study. Patients received monotherapy with navoximod 400 mg, $600 \mathrm{mg}$ or $1000 \mathrm{mg}$ orally twice daily (BID) in Stage 1 and navoximod $200 \mathrm{mg}, 400 \mathrm{mg}, 600 \mathrm{mg}$ or $1000 \mathrm{mg}$ orally BID plus atezolizumab $1200 \mathrm{mg}$ intravenously every 21 days in Stage 2. Objectives included safety, tolerability, efficacy and pharmacokinetic outcomes.Overall, 20 patients were enrolled (Stage 1: $n=10$; Stage 2: $n=10$ ). No dose-limiting toxicities were observed. In Stage 1, treatment-related adverse events (TRAEs) of any grade that occurred in $\geq 20 \%$ of patients were chromaturia ( $50 \%$ ) and maculopapular rash (20\%). Grade $\geq 3$ TRAEs were reported in two patients (20\%; maculopapular rash and lipase increased). In Stage 2, TRAEs that occurred in $\geq 30 \%$ of patients were chromaturia $(60 \%)$ and- decreased appetite (40\%). Grade $\geq 3$ TRAEs were reported in three patients (30\%; hyponatraemia, aspartate aminotransferase increased, alanine aminotransferase increased, lymphopaenia and neutropaenia). Stable disease was observed in five patients (50\%) in Stage 1 and eight patients (80\%) in Stage 2. Navoximod showed linear pharmacokinetics. The recommended dose of navoximod monotherapy was determined as $1000 \mathrm{mg}$ orally BID, and could be considered $1000 \mathrm{mg}$ orally BID in combination with atezolizumab. Navoximod as monotherapy and in combination with atezolizumab was well tolerated in Japanese patients with advanced solid tumours.

Keywords Atezolizumab · Cancer · GDC-0919 $\cdot$ Japanese $\cdot$ Navoximod $\cdot$ Phase I

Electronic supplementary material The online version of this article (https://doi.org/10.1007/s10637-019-00787-3) contains supplementary material, which is available to authorized users.

Noboru Yamamoto

nbryamam@ncc.go.jp

1 Department of Experimental Therapeutics, National Cancer Center Hospital, 5-1-1, Tsukiji, Chuo-ku, Tokyo 1040045, Japan

2 Department of Breast and Medical Oncology, National Cancer Center Hospital, Tokyo, Japan

3 Chugai Pharmaceutical Co., Ltd., Tokyo, Japan

\section{Introduction}

Indoleamine-2,3-dioxygenase 1 (IDO1) is an intracellular enzyme that catalyses the first, rate-limiting step in the kynurenine pathway of tryptophan catabolism [1]. IDO1 creates a tryptophan-depleted microenvironment, which induces a starvation response in $\mathrm{T}$ cells and promotes regulatory $\mathrm{T}$ cell (Treg) differentiation. In addition, kynurenine and other downstream products of IDO1 promote Treg differentiation and induce an immunosuppressive phenotype in dendritic cells and macrophages [2]. As a result, IDO1 has local and systemic immunosuppressant and tolerogenic effects [1].

IDO1 is expressed in multiple tumour types, including melanoma, pancreatic adenocarcinoma, ovarian cancer, acute myeloid leukaemia, colorectal cancer, prostate cancer and 
endometrial cancer [3-9]. The level of IDO1 expression has been shown to predict poor outcomes in these cancers [3-9]. Due to its role in tumour immunosuppression, IDO1 represents an attractive target for cancer therapy [10].

Navoximod (GDC-0919) is an investigational small-molecule inhibitor of IDO1 characterised by oral bioavailability and a favourable pharmacokinetic profile, as evaluated in animal models and human participants [11]. Navoximod has been shown to inhibit IDO1 in vitro in cell-based assays. Following oral administration, navoximod reduced plasma and tissue kynurenine concentrations by approximately $50 \%$, blocked IDO1-induced T cell suppression and restored $\mathrm{T}$ cell function [11].

The aim of this study was to evaluate the safety, tolerability and pharmacokinetics of navoximod alone and in combination with atezolizumab in Japanese patients with advanced solid tumours.

\section{Methods}

\section{Design and patients}

This was a phase I, open-label, single-centre, dose-escalation study conducted between August 2016 and February 2018 in Japanese patients with advanced solid tumours. The study consisted of two stages (Fig. 1). During Stage 1, to allow for pharmacokinetic evaluation, patients received a single initial dose of oral navoximod $400 \mathrm{mg}, 600 \mathrm{mg}$ or $1000 \mathrm{mg}$; 38 days after that, treatment with the same dose twice daily was initiated. Stage 2 was initiated after tolerability of the lowest dose of navoximod ( $400 \mathrm{mg}$ ) was confirmed in Stage 1. During Stage 2, patients received a single initial dose of oral navoximod $200 \mathrm{mg}, 400 \mathrm{mg}, 600 \mathrm{mg}$ or $1000 \mathrm{mg}$, followed by

Fig. 1 Study design

Stage 1

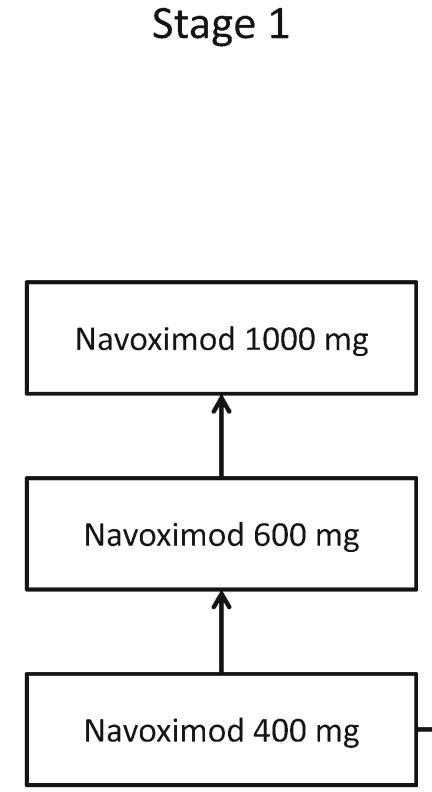

twice-daily navoximod plus intravenous atezolizumab $1200 \mathrm{mg}$ every 21 days.

The study protocol was approved by the institutional review boards of all participating centres and the study was conducted in accordance with the Declaration of Helsinki, Good Clinical Practice, and the Law for Ensuring the Quality, Efficacy, and Safety of Drugs and Medical Devices (paragraph 3 of article 14 and article 80-2). All study participants provided written informed consent before entering the study.

Patients were eligible for enrolment if they were aged $\geq 20$ years, had a histologically or cytologically confirmed advanced or recurrent solid tumour refractory to standard therapy or for which no standard therapy existed, an Eastern Cooperative Oncology Group (ECOG) performance status of 0 or 1 , life expectancy $\geq 12$ weeks, radiographically evaluable lesions and adequate haematological and major organ function. Patients were required to complete surgery, radiotherapy, antibody therapy (except anti-programmed cell death protein [PD-1]/programmed death-ligand 1 [PD-L1] and anti-cytotoxic $\mathrm{T}$ lymphocyte-associated antigen [CTLA]-4 drugs), immunosuppressive therapy, live vaccines and other investigational drugs within 4 weeks of enrolment; chemotherapy within 3 weeks; anti-PD-1/PD-L1 antibody drugs within 10 weeks; anti-CTLA-4 antibody drugs and other cancer immunotherapy within 6 weeks; and blood transfusion, hematopoietic growth factors and endocrine therapy within 2 weeks. The main exclusion criteria were a history of hypersensitivity to an excipient of navoximod; gastrointestinal condition that could interfere with drug absorption; central nervous system or meningeal metastases that were symptomatic or required treatment; pleural effusion, pericardial effusion or ascites requiring drainage; ongoing grade $\geq 2$ adverse reaction to a previous treatment (assessed according to

Stage 2

Navoximod $1000 \mathrm{mg}+$ Atezolizumab

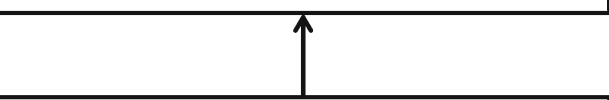

Navoximod $600 \mathrm{mg}+$ Atezolizumab

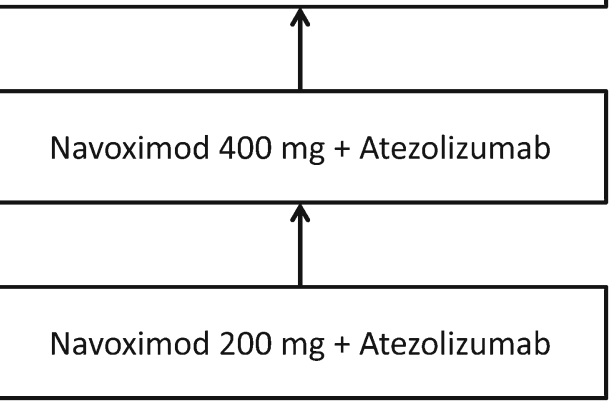


the National Cancer Institute Common Terminology Criteria for Adverse Events [NCI CTCAE] version 4.03); previous grade $\geq 3$ adverse reaction to an immunologically targeted anti-tumour drug; active infection requiring systemic treatment; current or previous autoimmune disease; current or previous clinically significant liver disease; and positive tests for human immunodeficiency virus, hepatitis B virus or hepatitis $\mathrm{C}$ virus. In addition, patients who had a history of hypersensitivity to drugs derived from Chinese hamster ovary cells or other recombinant human antibodies or recombinant proteins were excluded from Stage 2.

The target sample size was $6-18$ patients ( $2-3$ cohorts of $3-$ 6 patients each) for Stage 1 and 9-24 patients (3-4 cohorts of 3-6 patients each) for Stage 2. Recruitment of patients to each dose level was sequential using the standard $3+3$ study design. Patients could only be recruited to the next higher dose of navoximod if $<1$ patient developed dose-limiting toxicity (DLT) at the existing dose. At each dose level, treatment was continued until disease progression, DLT or other adverse event (AE) that would hinder the patient's participation in the study, or until the patient requested discontinuation from the study.

Plasma samples were collected for analysis of navoximod and kynurenine/tryptophan levels according to the schedule outlined in Supplementary Table 1.

\section{Outcomes}

Safety, pharmacokinetics, efficacy, exploratory pharmacodynamics and genetic outcomes were assessed in this study. The safety outcomes were the incidence, type, severity and time of onset of DLTs, AEs and treatment-related AEs (TRAEs). AEs were assessed using NCI CTCAE version 4.03. In addition, the maximum tolerated dose (MTD) was determined on the basis of DLTs. DLT was defined as one of the AEs listed in Supplementary Table 2 that occurred during the single dose administration period (2-7 days) and the first 21 days of twice daily navoximod administration in Stage 1 and Stage 2, and for which a potential causal relationship between navoximod and the AE could not be ruled out. MTD was defined as the highest dose at which $<33 \%$ of patients experienced a DLT.

The pharmacokinetic outcomes were the plasma concentration of navoximod, area under the plasma concentration-time curve (AUC), peak plasma concentration $\left(\mathrm{C}_{\max }\right)$, time to $\mathrm{C}_{\max }\left(\mathrm{T}_{\max }\right)$ and plasma elimination half-life $\left(t_{1 / 2}\right)$. The efficacy outcomes were the response rate, disease control rate, progression-free survival (PFS) and duration of response. Efficacy outcomes were assessed using the Response Evaluation Criteria in Solid Tumours (RECIST) version 1.1. Plasma concentrations of kynurenine and tryptophan and their ratios and the presence of UGT1A $1 * 6$ and UGT1A $1 * 28$ polymorphisms were the exploratory outcomes of this study.

\section{Statistics}

Safety endpoints were analysed in the safety population, which consisted of patients who received $\geq 1$ dose of the study drug. Efficacy endpoints were analysed in the full analysis set, which consisted of patients who received $\geq 1$ dose of the study drug and underwent $\geq 1$ post-baseline efficacy assessment. A summary of AEs, TRAEs and DLTs by mapped term, appropriate thesaurus level and severity was provided for each cohort. Summary statistics, including mean, standard deviation, coefficient of variation, median, and minimum and maximum, were calculated for pharmacokinetic and pharmacodynamic parameters.

\section{Results}

Between 31 August 2016 and 30 May 2017, 20 patients were enrolled and received navoximod (10 patients in Stage 1 and 10 patients in Stage 2; Table 1). Enrolment in Stage 2 was discontinued earlier than planned and only one patient was enrolled in the navoximod $1000 \mathrm{mg}$ cohort. Patients were aged between 48 and 75 years. Diagnoses included thymic cancer, pancreatic cancer, small-cell lung cancer, adenoid cystic cancer of the palatal gingiva, leiomyosarcoma, cervical cancer, endometrial cancer, olfactory neuroblastoma, nonsmall-cell lung cancer, peritoneal cancer, ovarian cancer and bladder cancer. Most patients had ECOG performance status of 0 at baseline $(n=13,65 \%)$.

\section{Safety}

During Stage 1, TRAEs were reported in six out of 10 patients (60\%; Table 2). Grade 3 TRAEs were reported in one patient (10\%) who received navoximod $400 \mathrm{mg}$ (maculopapular rash) and one patient (10\%) who received navoximod $600 \mathrm{mg}$ (lipase increased). The latter TRAE did not resolve after navoximod treatment was suspended, however, there were no other symptoms or abnormal findings. No grade 4 or 5 TRAEs were observed. In addition, no DLTs were observed during Stage 1 and the MTD was not reached. Based on these results, the recommended dose of navoximod monotherapy was determined as $1000 \mathrm{mg}$ orally twice daily.

During Stage 2, TRAEs were reported in all 10 patients (100\%; Table 3). Grade 3 TRAEs were reported in three patients $(30 \%)$ and included hyponatraemia, lymphopenia, neutropenia and elevated AST and ALT. All grade 3 TRAEs were confirmed to have resolved. No grade 4 or 5 TRAEs were observed. During Stage 2, no DLTs were observed and the MTD was not reached. The recommended dose of navoximod in combination with atezolizumab was not determined because of early discontinuation; however, $1000 \mathrm{mg}$ orally twice daily was well tolerated. 
Table 1 Baseline patient characteristics

Stage 1

\begin{tabular}{llll}
\hline Navoximod & Navoximod & Navoximod & Stage 1 \\
$400 \mathrm{mg}$ & $600 \mathrm{mg}$ & $1000 \mathrm{mg}$ & total \\
$(N=3)$ & $(N=4)$ & $(N=3)$ & $(N=10)$
\end{tabular}

Stage 2

\begin{tabular}{lllll}
\hline Navoximod & Navoximod & Navoximod & Navoximod & Stage 2 \\
$200 \mathrm{mg}+$ & $400 \mathrm{mg}+$ & $600 \mathrm{mg}+$ & $1000 \mathrm{mg}+$ & total \\
Atezolizumab & Atezolizumab & Atezolizumab & Atezolizumab & $(N=10)$ \\
$(N=3)$ & $(N=3)$ & $(N=3)$ & $(N=1)$ &
\end{tabular}

\section{Age, years}

$\begin{array}{llllllllll}\text { Median } & 68 & 62 & 54 & 61.5 & 58 & 68 & 66 & 58 & 63.5 \\ \text { Range } & 64-74 & 48-75 & 52-59 & 48-75 & 57-70 & 56-70 & 61-69 & - & 56-70\end{array}$

Sex, $n$

Male 2

Female 1

$2 \quad 0$

ECOG PS, $n$

$0 \quad 0$

13

3

No. of prior systemic treatments, $\mathrm{n}$

\begin{tabular}{lllll} 
Median & 2 & 4 & 4 & 3 \\
Range & $2-3$ & $2-6$ & $1-11$ & $1-11$ \\
\hline
\end{tabular}

ECOG Eastern Cooperative Oncology Group, $P S$ performance status

\section{Pharmacokinetics}

After a single oral dose of navoximod, administered as monotherapy (Stage 1) or in combination with atezolizumab (Stage 2 ), the mean plasma concentration peaked at 15-60 min after administration and decreased precipitously after that (Fig. 2). When navoximod was administered alone in Stage 1, AUC and $\mathrm{C}_{\max }$ changed dose-proportionally in the $400 \mathrm{mg}, 600 \mathrm{mg}$ and $1000 \mathrm{mg}$ cohorts. Similar results were obtained when navoximod was administered in combination with atezolizumab in Stage 2.

Analysis of variance did not produce any statistically significant results. In linear regression analysis, the $95 \%$ confidence interval $(95 \% \mathrm{CI})$ for the intercept of dose exposure contained 0 and the $95 \%$ CI for the intercept of the power model contained 1 (Fig. 3).
Dose-corrected navoximod exposure was similar in patients with UGT1A1 -/-, UGT1A1 -/*6, and UGT1A1 *6/ *6; however, dose-corrected exposure was higher in patients with UGT1A1 -/*28.

The change from baseline in kynurenine/tryptophan ratio was more marked with increasing doses of navoximod (Fig. 4).

\section{Efficacy}

Duration of treatment by cancer type in Stage 1 and Stage 2 are shown in Fig. 5a and b, respectively, along with the key reasons for navoximod discontinuation.

During Stage 1, best overall response was stable disease (SD) in five patients (navoximod $600 \mathrm{mg}$ : $n=2$; navoximod $1000 \mathrm{mg}: n=3$ ) and progressive disease (PD) in five patients (navoximod $400 \mathrm{mg}$ : $n=3$; navoximod $600 \mathrm{mg}$ : $n=2$;
Table 2 Treatment-related adverse events reported in two or more patients during Stage 1

\begin{tabular}{|c|c|c|c|c|}
\hline TRAE, $n$ & $\begin{array}{l}\text { Navoximod } \\
400 \mathrm{mg}(N=3)\end{array}$ & $\begin{array}{l}\text { Navoximod } \\
600 \mathrm{mg}(N=4)\end{array}$ & $\begin{array}{l}\text { Navoximod } \\
1000 \mathrm{mg}(N=3)\end{array}$ & $\begin{array}{l}\text { Total } \\
(N=10)\end{array}$ \\
\hline \multicolumn{5}{|l|}{ Any, $\mathrm{n}$} \\
\hline All grades & 1 & 2 & 3 & 6 \\
\hline Grade $\geq 3$ & 1 & 1 & 0 & 2 \\
\hline \multicolumn{5}{|c|}{ Chromaturia, $n$} \\
\hline All grades & 0 & 2 & 3 & 5 \\
\hline Grade $\geq 3$ & 0 & 0 & 0 & 0 \\
\hline \multicolumn{5}{|c|}{ Maculopapular rash, $\mathrm{n}$} \\
\hline All grades & 1 & 0 & 1 & 2 \\
\hline Grade $\geq 3$ & 1 & 0 & 0 & 1 \\
\hline
\end{tabular}

TRAE treatment-related adverse event 
Table 3 Treatment-related adverse events reported in two or more patients during Stage 2

\begin{tabular}{|c|c|c|c|c|c|}
\hline TRAE, $n$ & $\begin{array}{l}\text { Navoximod } \\
200 \mathrm{mg}+ \\
\text { Atezolizumab } \\
(N=3)\end{array}$ & $\begin{array}{l}\text { Navoximod } \\
400 \mathrm{mg}+ \\
\text { Atezolizumab } \\
(N=3)\end{array}$ & $\begin{array}{l}\text { Navoximod } \\
600 \mathrm{mg}+ \\
\text { Atezolizumab } \\
(N=3)\end{array}$ & $\begin{array}{l}\text { Navoximod } \\
1000 \mathrm{mg}+ \\
\text { Atezolizumab } \\
(N=1)\end{array}$ & $\begin{array}{l}\text { Total } \\
(N=10)\end{array}$ \\
\hline \multicolumn{6}{|l|}{ Any } \\
\hline All grades & 3 & 3 & 3 & 1 & 10 \\
\hline Grade $\geq 3$ & 1 & 2 & 0 & 0 & 3 \\
\hline \multicolumn{6}{|l|}{ Fatigue } \\
\hline All grades & 1 & 0 & 1 & 0 & 2 \\
\hline Grade $\geq 3$ & 0 & 0 & 0 & 0 & 0 \\
\hline \multicolumn{6}{|l|}{ Chromaturia } \\
\hline All grades & 2 & 0 & 3 & 1 & 6 \\
\hline Grade $\geq 3$ & 0 & 0 & 0 & 0 & 0 \\
\hline \multicolumn{6}{|c|}{ Decreased appetite } \\
\hline All grades & 1 & 1 & 1 & 0 & 3 \\
\hline Grade $\geq 3$ & 0 & 0 & 0 & 0 & 0 \\
\hline \multicolumn{6}{|c|}{ Hyponatraemia } \\
\hline All grades & 1 & 1 & 0 & 0 & 2 \\
\hline Grade $\geq 3$ & 1 & 1 & 0 & 0 & 2 \\
\hline \multicolumn{6}{|c|}{ AST increased } \\
\hline All grades & 0 & 2 & 0 & 0 & 2 \\
\hline Grade $\geq 3$ & 0 & 1 & 0 & 0 & 1 \\
\hline \multicolumn{6}{|c|}{ ALT increased } \\
\hline All grades & 0 & 2 & 0 & 0 & 2 \\
\hline Grade $\geq 3$ & 0 & 1 & 0 & 0 & 1 \\
\hline
\end{tabular}

$A L T$ alanine aminotransferase, $A S T$ aspartate aminotransferase, $T R A E$ treatment-related adverse event
Fig. 6a). Complete response (CR) and partial response (PR) were not observed in any of the patients during Stage 1.
Disease control was achieved in four out of 10 patients (40\%). PFS ranged from 9 to 259 days.
Fig. 2 Plasma concentration of navoximod over time after single oral dose

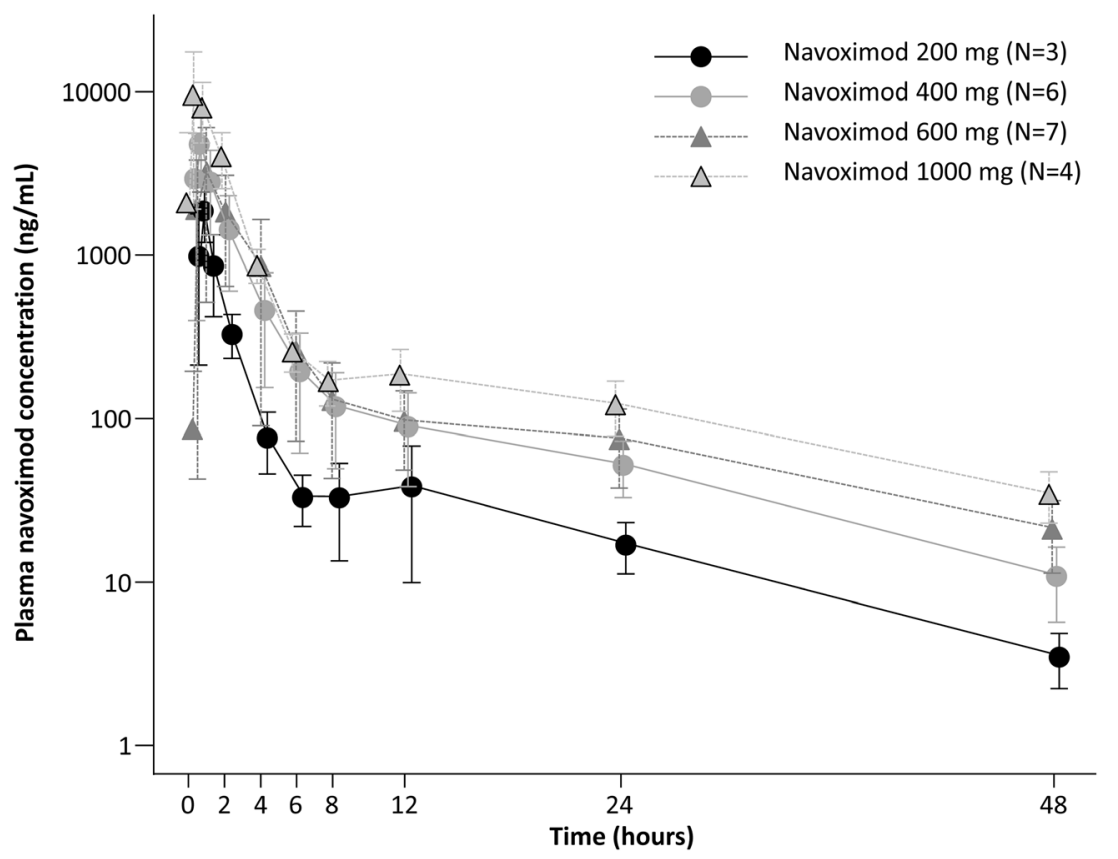


Fig. 3 AUC after a single oral dose of navoximod AUC, area under the plasma concentrationtime curve

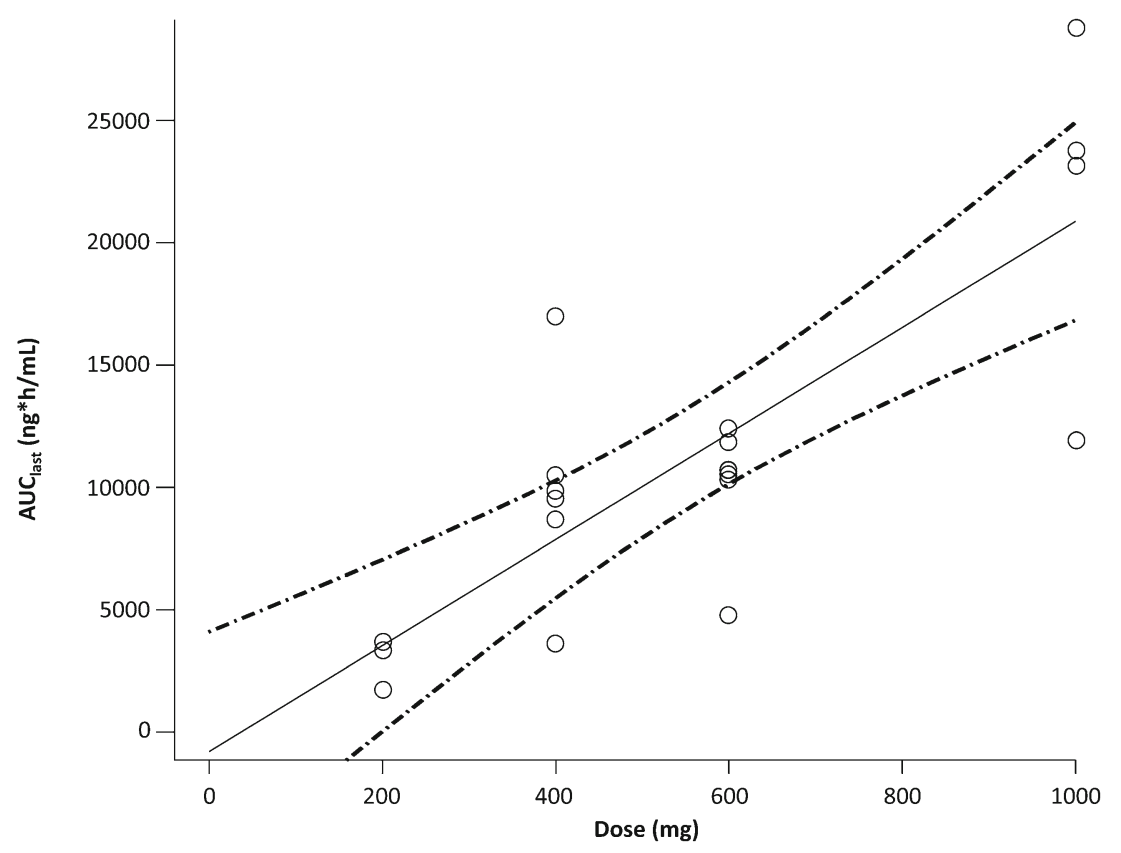

During Stage 2, best overall response was SD in eight patients (navoximod $200 \mathrm{mg}+$ atezolizumab: $n=3$; navoximod $400 \mathrm{mg}+$ atezolizumab: $n=2$; navoximod $600 \mathrm{mg}$ + atezolizumab: $n=2$; navoximod $1000 \mathrm{mg}+$ atezolizumab: $n=1$ ) and PD in two patients (navoximod $400 \mathrm{mg}$ + atezolizumab: $n=1$; navoximod $600 \mathrm{mg}+$ atezolizumab: $n=1$; Fig. 6 b). None of the patients had CR or PR during Stage 2. Disease control was achieved in eight out of 10 patients (80\%). PFS ranged from 19 to 339 days.

\section{Discussion}

The results of this phase I, open-label, single-centre, doseescalation study indicate that navoximod, alone or in combination with atezolizumab, was well tolerated in Japanese patients with advanced solid tumours. No DLTs were observed during this study and MTD was not reached. As a result, the recommended dose of navoximod monotherapy was determined as $1000 \mathrm{mg}$ twice daily. In combination with
Fig. 4 Percent change in plasma kynurenine-tryptophan ratio after single oral dose of navoximod

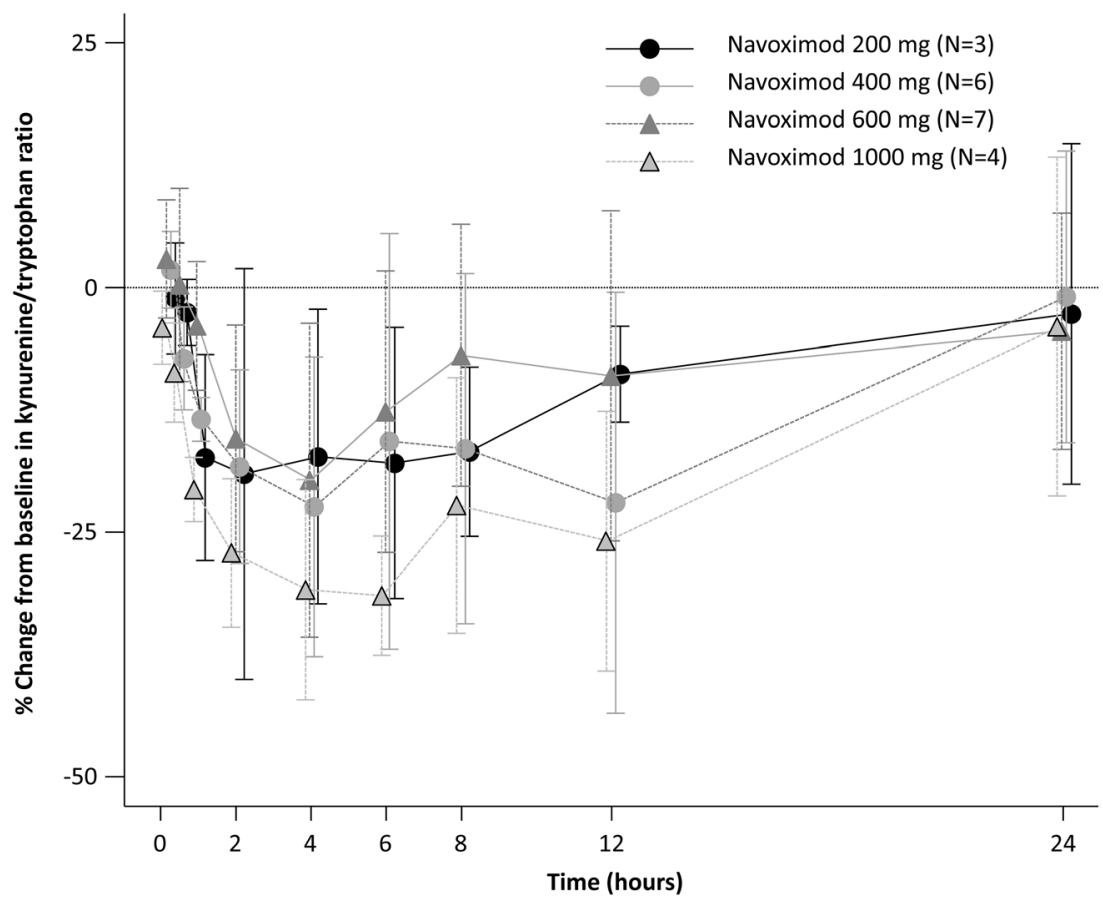


Fig. 5 Time on treatment in a Stage 1; b Stage 2 ID,

investigator's decision; NSCLC, non-small-cell lung cancer; PD, progressive disease; SCLC, small-cell lung cancer; NC, noncompliant to the study treatment after being informed about discontinuation of navoximod development

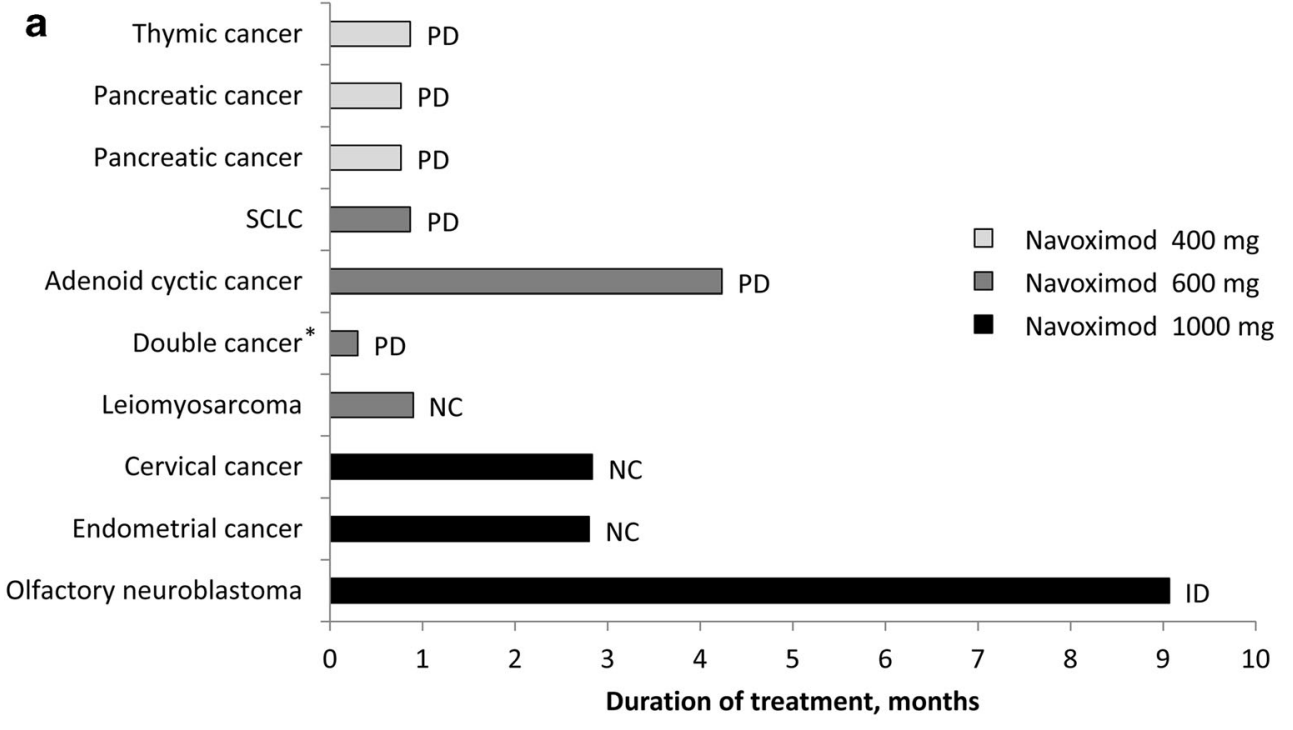

*Unknown primary cancer or thyroid cancer

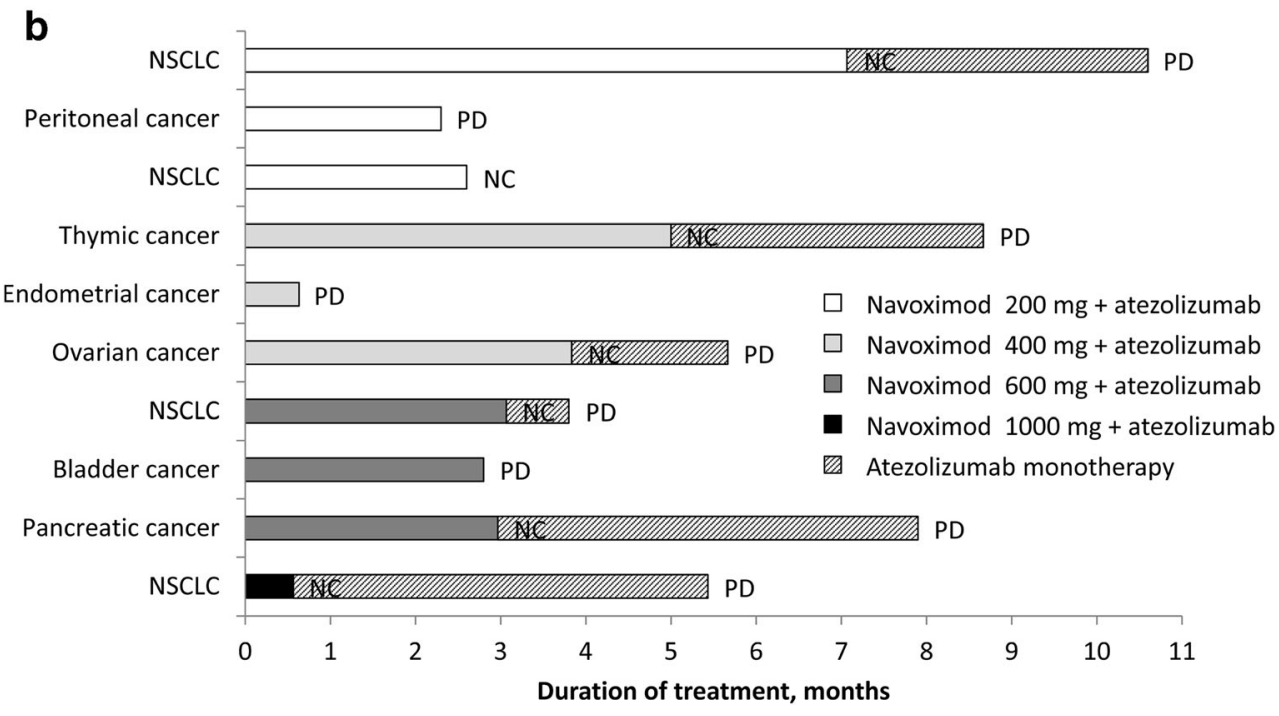

atezolizumab, the recommended dose of navoximod could not been determined because of early discontinuation of enrollment based on limited evidence of clinical activity in the clinical study conducted for the same time [12]. However, even though navoximod $1000 \mathrm{mg}$ twice daily in combination with atezolizumab was administered in one patient, there was no safety concern, as such the navoximod $1000 \mathrm{mg}$ twice daily in combination with atezolizumab $1200 \mathrm{mg}$ every 21 days could be considered as recommended dose in our study.

The only symptomatic grade 3 or higher TRAE observed in this study was maculopapular rash $(5 \%)$. The incidence of chromaturia was relatively high $(55 \%)$; however, all events were asymptomatic. The mechanisms responsible for the development of chromaturia are not fully elucidated. Navoximod peak plasma concentration and exposure were dose-proportional in the $200-1000 \mathrm{mg}$ range. In the present study, no PRs or CRs were observed with either navoximod monotherapy or navoximod plus atezolizumab combination. However, the majority of patients in both Stage 1 and Stage 2 had a best overall response of SD. Modest IDO1 inhibitory activity was observed with navoximod in the present study, as evidenced by reductions in the kynurenine/tryptophan ratio of up to approximately $50 \%$. In the $200-1000 \mathrm{mg}$ range, this activity increased in a dose-proportional manner.

The findings of this study are in line with those of the firstin-human study of navoximod monotherapy [13], and the combination study of navoximod and atezolizumab [12]. In the monotherapy study, 22 patients with advanced or recurrent solid tumours received monotherapy with navoximod $100 \mathrm{mg}, 200 \mathrm{mg}, 400 \mathrm{mg}, 600 \mathrm{mg}$ or $800 \mathrm{mg}$ twice daily for 21 days, followed by 7 days without treatment, or $600 \mathrm{mg}$ twice daily continuously. As in the present study, MTD was 
Fig. 6 Best percent change from baseline in a Stage 1 ; b Stage 2 $\mathrm{PD}$, progressive disease; $\mathrm{SD}$, stable disease

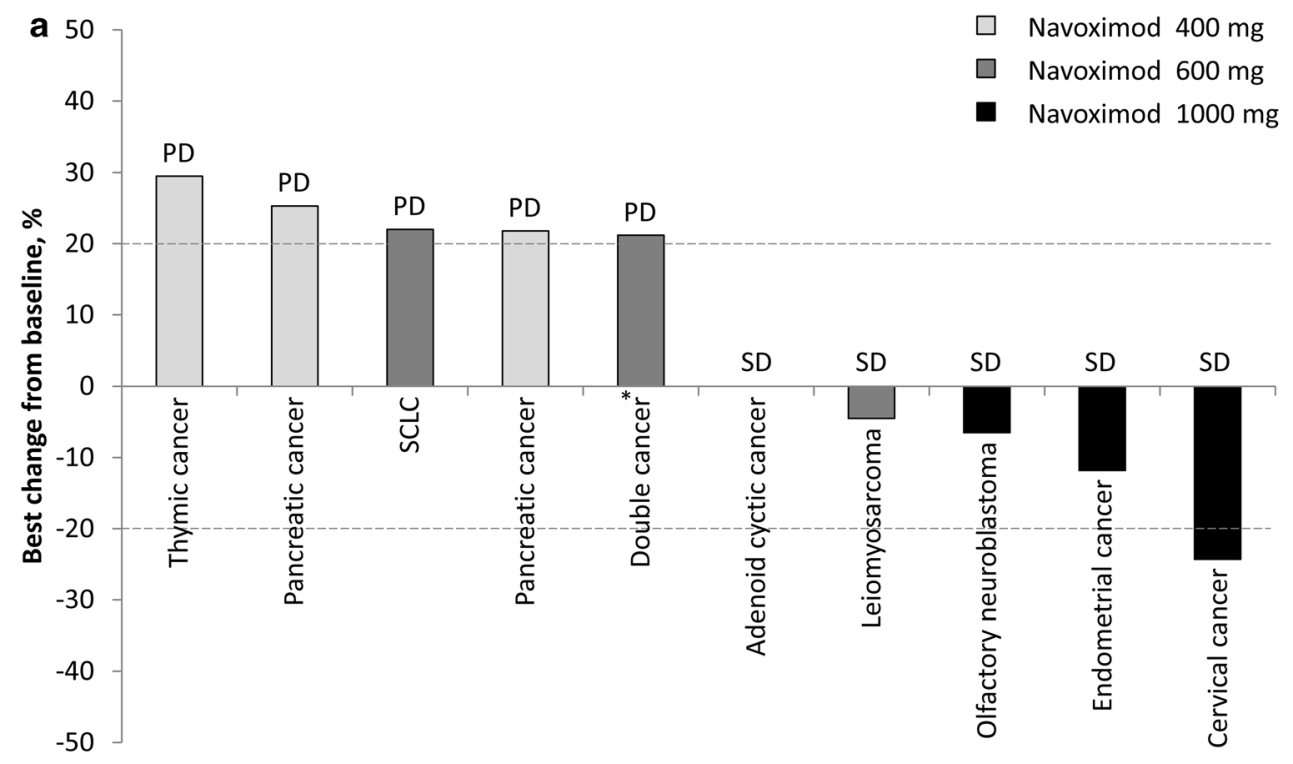

*Unknown primary cancer or thyroid cancer

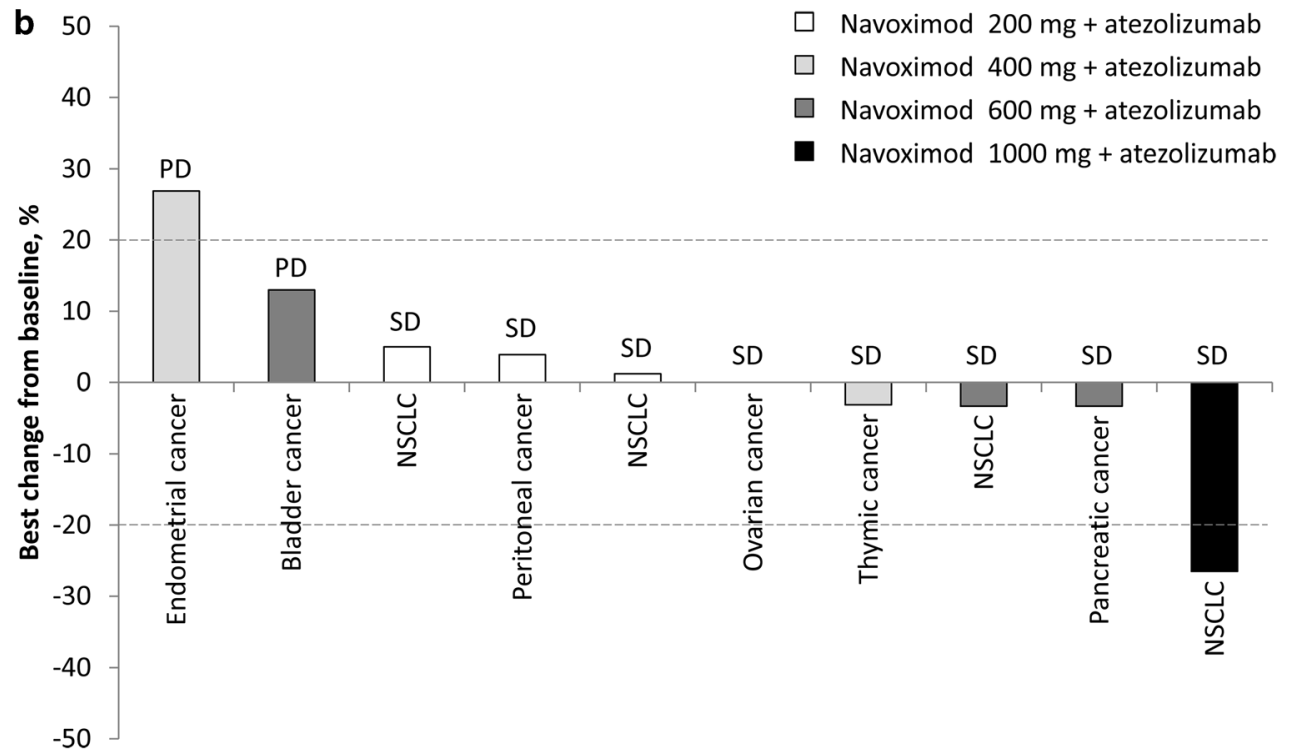

not reached. Grade 3 or higher AEs occurred in two patients (9\%; lower gastrointestinal haemorrhage, diverticulitis), and dose interruptions and/or reductions were required in two patients. Similar to the present study, navoximod demonstrated linear pharmacokinetics with time to peak plasma concentration of approximately $60 \mathrm{~min}$ and a half-life of approximately $12 \mathrm{~h}$. As in the present study, no CRs or PRs were observed. In patients who received navoximod $400 \mathrm{mg}, 600 \mathrm{mg}$ or $800 \mathrm{mg}$, plasma kynurenine levels decreased by approximately 25 $30 \%$ at $2-4 \mathrm{~h}$ after administration [13]. In the combination study, 157 patients with advanced or recurrent solid tumours received navoximod at 6 dose levels (50-1000 mg twice daily continuously) in combination with atezolizumab. There was a single DLT of grade 3 sepsis syndrome at the $200 \mathrm{mg}$ dose level. The tolerability of navoximod $1000 \mathrm{mg}$ in combination with atezolizumab was confirmed and MTD was not reached. Grade 3 or higher treatment-related AEs occurred in 3 or more patients were rash (9\%), fatigue (2\%) and hepatitis (2\%). The pharmacokinetics of navoximod in combination with atezolizumab was consistent with the pharmacokinetic observed in monotherapy. Sixteen patients $(20 \%)$ achieved partial response or complete response. The administration of navoximod decreased plasma kynurenine: navoximod $1000 \mathrm{mg}$ exceeded the IC50 of IDO1 in $\sim 90 \%$ of the patients 
and decreases approximately $25 \%$ of plasma kynurenine. The efficacy of kynurenine modulation in this study was not so different from that of the combination study and as such, the recommended dose of navoximod in combination with atezolizumab based on the results of this study could be considered equal to that observed in the navoximod combination study.

Although navoximod was well tolerated in the present study and the monotherapy and combination therapy studies $[12,13]$, the efficacy of navoximod was modest. Previous studies with other IDO1 inhibitors have shown mixed efficacy results. In a phase I/II study in patients with advanced solid tumours, epacadostat plus nivolumab was associated with PR in some patients [14]. In a phase I/IIa study conducted in 29 patients with advanced bladder cancer, the combination of linrodostat plus nivolumab was associated with $\mathrm{CR}$ in one patient and PR in nine patients, resulting in the overall response rate of 34\% [15]. Despite promising results of the phase I/II study, epacadostat in combination with pembrolizumab failed to show efficacy in a phase III, randomised, double-blind, placebo-controlled study conducted in 706 patients with unresectable or metastatic melanoma (ECHO-301/KEYNOTE-252) [16]. There were no significant differences between epacadostat plus pembrolizumab and placebo plus pembrolizumab in either PFS (hazard ratio $[\mathrm{HR}]=$ $1.00 ; 95 \%$ CI $0.83-1.21 ; p=0.517)$ or overall survival (HR = $1.13 ; 95 \%$ CI $0.86-1.49 ; p=0.807)$ [16]. Insufficient inhibition of kynurenine synthesis, inappropriate patient selection and crosstalk between PD-1/PD-L1 and IDO1 pathways could have contributed to these results.

Discovery of IDO1 stimulated research into the immunomodulatory effects of tryptophan, kynurenine and other metabolites of the kynurenine pathway, and this has improved our understanding of the immune system $[1,17]$. However, preclinical studies have shown that IDO1 inhibitors are ineffective as stand-alone cancer treatments [13]. It should be remembered that inhibitors of PD-1 and PD-L1 have also shown modest activity when used as monotherapy. Due to the complexity of the immune system, it is likely that combinations of two or more immune checkpoint inhibitors will be necessary for effective cancer therapy [13]. Therefore, the therapeutic role of IDO1 inhibitors is likely to be as part of a combination regimen, rather than as monotherapy.

In conclusion, navoximod was well tolerated as monotherapy and in combination with atezolizumab in Japanese patients with advanced solid tumours. Additional research into the role of the IDO1 pathway in the maintenance of tumour microenvironment is necessary for further development of IDO1 inhibitors.

Acknowledgments We would like to thank Georgii Filatov of Springer Healthcare Communications who wrote the first draft and subsequent drafts of this manuscript. This medical writing assistance was funded by Chugai Pharmaceutical Co., Ltd.
Authors' contributions Yamamoto N. contributed to the study design, enrolment of patients, drafting the manuscript, and reviewing the manuscript. Ebata T contributed to the enrolment of patients and drafting the manuscript. Nakai K. and Inatani M. contributed to the study design, analysis of data and reviewed the manuscript. All other authors were involved in patient enrolment, and reviewed and approved the manuscript at each stage and before submission.

Funding The work was supported by Chugai Pharmaceutical Co., Ltd. in Tokyo, Japan.

\section{Compliance with ethical standards}

Conflict of interest Ebata $\mathrm{T}$ declares that he has no conflict of interest. Kondo $\mathrm{S}$ declares that he has no conflict of interest. Yonemori $\mathrm{K}$ declares that he has no conflict of interest. Shimomura A declares that he has no conflict of interest. Koyama $\mathrm{T}$ declares that he has no conflict of interest. Yamamoto $\mathrm{N}$ is an advisory board member for Eisai, Takeda Pharmaceutical, Otsuka Pharmaceutical, Boehringer Ingelheim, and Cmic Pharma Science, has been involved with sponsored research for Eli Lilly, Abbvie, Janssen Pharmaceutical, MSD, Merck, Quintiles, Astellas Pharma, Chugai Pharmaceutical Co. Ltd., Esai, Taiho, Bristol-Myers Squibb, Pfizer, Novartis Pharma, Daiichi Sankyo, Bayer, Boehringer Ingelheim, Kyowa Hakko Kirin, Takeda Pharmaceutical, and Ono Pharmaceutical, and has received lecture fees from Bristol-Myers Squibb, Pfizer, AstraZeneca, Eli Lilly, Ono Pharmaceutical, and Chugai Pharmaceutical. Shimizu T. has been involved with sponsored research for Bristol-Myers Squibb. Fujiwara Y. is an advisory board member for Bristol-Myers Squibb, Takeda Pharmaceutical, and The Consortium on Harmonization of Institutional Requirements for Clinical Research (CHAIR), Hong Kong, HKSAR and has been involved with sponsored research for Chugai Pharmaceutical Co. Ltd., Incyte Corporation, MSD, Bristol-Myers Squibb, Daiichi Sankyo, Eli Lilly, Novartis Pharma, Eisai, Millenium-Takeda Pharmaceutical, PharmaMar, Five Prime, 3D-Medicine, SymBio Pharmaceuticals and Chordia Therapeutics. Tamura $\mathrm{K}$ is an advisory board member for MSD and has been involved with sponsored research for Chugai Pharmaceutical, Bristol-Myers Squibb and MSD. Iwasa S. has been involved with sponsored research for Bristol-Myers Squibb and Chugai Pharmaceutical. Kitano S is an advisory board member for BristolMyers Squibb and MSD. Sato N, Nakai K, and Inatani M. are employees of Chugai Pharmaceutical.

Ethical approval All procedures performed in studies involving human participants were in accordance with the ethical standards of the institutional and/or national research committee and with the 1964 Helsinki declaration and its later amendments or comparable ethical standards. The study protocol was approved by the Institutional Review Boards of the participating institution.

Informed consent Informed consent was obtained from all individual participants included in the study.

Open Access This article is distributed under the terms of the Creative Commons Attribution 4.0 International License (http:// creativecommons.org/licenses/by/4.0/), which permits unrestricted use, distribution, and reproduction in any medium, provided you give appropriate credit to the original author(s) and the source, provide a link to the Creative Commons license, and indicate if changes were made. 


\section{References}

1. Munn DH, Mellor AL (2016) IDO in the tumor microenvironment: inflammation, counter-regulation, and tolerance. Trends Immunol 37:193-207. https://doi.org/10.1016/j.it.2016.01.002

2. Holmgaard RB, Zamarin D, Li Y, Gasmi B, Munn DH, Allison JP, Merghoub T, Wolchok JD (2015) Tumor-expressed IDO recruits and activates MDSCs in a Treg-dependent manner. Cell Rep 13: 412-424. https://doi.org/10.1016/j.celrep.2015.08.077

3. Brody JR, Costantino CL, Berger AC, Sato T, Lisanti MP, Yeo CJ, Emmons RV, Witkiewicz AK (2009) Expression of indoleamine 2, 3-dioxygenase in metastatic malignant melanoma recruits regulatory $\mathrm{T}$ cells to avoid immune detection and affects survival. Cell Cycle 8:1930-1934. https://doi.org/10.4161/cc.8.12.8745

4. Witkiewicz A, Williams TK, Cozzitorto J, Durkan B, Showalter SL, Yeo CJ, Brody JR (2008) Expression of indoleamine 2,3dioxygenase in metastatic pancreatic ductal adenocarcinoma recruits regulatory $\mathrm{T}$ cells to avoid immune detection. J Am Coll Surg 206:849-854; discussion 854-846. https://doi.org/10.1016/j. jamcollsurg.2007.12.014

5. Okamoto A, Nikaido T, Ochiai K, Takakura S, Saito M, Aoki Y, Ishii N, Yanaihara N, Yamada K, Takikawa O, Kawaguchi R, Isonishi S, Tanaka T, Urashima M (2005) Indoleamine 2,3dioxygenase serves as a marker of poor prognosis in gene expression profiles of serous ovarian cancer cells. Clin Cancer Res 11: 6030-6039. https://doi.org/10.1158/1078-0432.CCR-04-2671

6. Chamuleau ME, van de Loosdrecht AA, Hess CJ, Janssen JJ, Zevenbergen A, Delwel R, Valk PJ, Lowenberg B, Ossenkoppele GJ (2008) High INDO (indoleamine 2,3-dioxygenase) mRNA level in blasts of acute myeloid leukemic patients predicts poor clinical outcome. Haematologica 93:1894-1898. https://doi.org/10.3324/ haematol.13113

7. Brandacher G, Perathoner A, Ladurner R, Schneeberger S, Obrist P, Winkler C, Werner ER, Werner-Felmayer G, Weiss HG, Gobel G, Margreiter R, Konigsrainer A, Fuchs D, Amberger A (2006) Prognostic value of indoleamine 2,3-dioxygenase expression in colorectal cancer: effect on tumor-infiltrating T cells. Clin Cancer Res 12:1144-1151. https://doi.org/10.1158/1078-0432.CCR-05-1966

8. Feder-Mengus C, Wyler S, Hudolin T, Ruszat R, Bubendorf L, Chiarugi A, Pittelli M, Weber WP, Bachmann A, Gasser TC, Sulser T, Heberer M, Spagnoli GC, Provenzano M (2008) High expression of indoleamine 2,3-dioxygenase gene in prostate cancer. Eur J Cancer 44:2266-2275. https://doi.org/10.1016/j.ejca.2008. 05.023

9. Ino K, Yamamoto E, Shibata K, Kajiyama H, Yoshida N, Terauchi M, Nawa A, Nagasaka T, Takikawa O, Kikkawa F (2008) Inverse correlation between tumoral indoleamine 2,3-dioxygenase expression and tumor-infiltrating lymphocytes in endometrial cancer: its association with disease progression and survival. Clin Cancer Res 14:2310-2317. https://doi.org/10.1158/1078-0432.CCR-07-4144

10. Prendergast GC, Smith C, Thomas S, Mandik-Nayak L, LauryKleintop L, Metz R, Muller AJ (2014) Indoleamine 2,3dioxygenase pathways of pathogenic inflammation and immune escape in cancer. Cancer Immunol Immunother 63:721-735. https://doi.org/10.1007/s00262-014-1549-4

11. Mautino MR, Jaipuri FA, Waldo J, Kumar S, Adams J, Van Allen C, Marcinowicz-Flick A, Munn D, Vahanian N, Link CJ (2013)
Abstract 491: NLG919, a novel indoleamine-2,3-dioxygenase (IDO)-pathway inhibitor drug candidate for cancer therapy. Cancer Res 73:491-491. https://doi.org/10.1158/1538-7445. am2013-491

12. Jung KH, LoRusso P, Burris H, Gordon M, Bang YJ, Hellmann MD, Cervantes A, de Olza MO, Marabelle A, Hodi FS, Ahn MJ, Emens LA, Barlesi F, Hamid O, Calvo E, McDermott D, Soliman H, Rhee I, Lin R, Pourmohamad T, Suchomel J, Tsuhako A, Morrissey K, Mahrus S, Morley R, Pirzkall A, Davis SL (2019) Phase I study of the indoleamine 2,3-dioxygenase 1 (IDO1) inhibitor navoximod (GDC-0919) administered with PD-L1 inhibitor (atezolizumab) in advanced solid tumors. Clin Cancer Res. https:// doi.org/10.1158/1078-0432.CCR-18-2740

13. Nayak-Kapoor A, Hao Z, Sadek R, Dobbins R, Marshall L, Vahanian NN, Jay Ramsey W, Kennedy E, Mautino MR, Link CJ, Lin RS, Royer-Joo S, Liang X, Salphati L, Morrissey KM, Mahrus S, McCall B, Pirzkall A, Munn DH, Janik JE, Khleif SN (2018) Phase Ia study of the indoleamine 2,3-dioxygenase 1 (IDO1) inhibitor navoximod (GDC-0919) in patients with recurrent advanced solid tumors. J Immunother Cancer 6:61. https://doi.org/ 10.1186/s40425-018-0351-9

14. Perez RP, Riese MJ, Lewis KD, Saleh MN, Daud A, Berlin J, Lee JJ, Mukhopadhyay S, Zhou L, Serbest G, Hamid O (2017) Epacadostat plus nivolumab in patients with advanced solid tumors: preliminary phase I/II results of ECHO-204. ASCO Meeting Library. https://meetinglibrary.asco.org/record/153930/abstract. Accessed 8 Nov 2018

15. Tabernero J, Luke JJ, Joshua AM, Varga AI, Moreno V, Desai J, Markman B, Gomez-Roca CA, Braud FGD, Patel SP, Carlino MS, Siu LL, Curigliano G, Liu Z, Ishii Y, Phillips P, Wind-Rotolo M, Basciano PA, Azrilevich A, Gelmon KA (2018) BMS-986205, an indoleamine 2,3-dioxygenase 1 inhibitor (IDO1i), in combination with nivolumab (NIVO): updated safety across all tumor cohorts and efficacy in pts with advanced bladder cancer (advBC). ASCO Meeting Library. https://meetinglibrary.asco.org/record/160571/ abstract. Accessed 8 Nov 2018

16. Long GV, Dummer R, Hamid O, Gajewski T, Caglevic C, Dalle S, Arance A, Carlino MS, Grob J-J, Kim TM, Demidov LV, Robert C, Larkin JMG, Anderson J, Maleski JE, Jones MM, Diede SJ, Mitchell TC (2018) Epacadostat (E) plus pembrolizumab (P) versus pembrolizumab alone in patients (pts) with unresectable or metastatic melanoma: results of the phase 3 ECHO-301/KEYNOTE252 study. ASCO Meeting Library. https://meetinglibrary.asco. org/record/164607/abstract. Accessed 8 Nov 2018

17. Platten M, von Knebel Doeberitz N, Oezen I, Wick W, Ochs K (2014) Cancer immunotherapy by targeting IDO1/TDO and their downstream effectors. Front Immunol 5:673. https://doi.org/10. 3389/fimmu.2014.00673

Publisher's note Springer Nature remains neutral with regard to jurisdictional claims in published maps and institutional affiliations. 